\title{
Pedicle Screw Fixation Applying Allogenic Bone Graft Augmentation in Patients with Severe Osteoporosis
}

\author{
Andrey Bokov*, Ivan Brattsev, Anatoliy Bulkin, Andrey Dydykin and Sergey Mlyavykh \\ Privolzhsky Research Medical University, Nizhny Novgorod, Russia
}

Submission: August 28, 2020; Published: September 15, 2020

*Corresponding author: Andrey Bokov, Privolzhsky Research Medical University, Nizhny Novgorod, Russia

Abstract

Objective: Objective of this study is to suggest an alternative to augmentation applying liquid substances and to study the results of pedicle screw fixation using allogeneic bone augmentation in patients with severe osteoporosis.

Methods: A prospective non-randomized study of 22 patients with either unstable traumatic injuries of lumbar spine or thoracolumbar junction or spinal stenosis with unstable segments with Tcr by DXA below $-3,5$ and radiodensity below $80 \mathrm{HU}$. Patients were treated employing pedicle screw fixation with allogeneic bone augmentation. Minimal follow-up period accounted for 18 months. Cases with implant instability were registered and analyzed.

Results: During the follow-up period CT signs of screw loosening were detected in 3. Out of those 2 patients presented with anterior or posterior fusion verified on CT therefore screw loosening was considered clinically insignificant. One patient presented with implant instability caused by wiper wind shield effect instability mechanism and revision pedicle screw was performed. The suggested technique provided favorable results in patients with severe osteoporosis with screw loosening detected on CT accounted for $13,6,95 \%$ CI $[2,9 ; 34,9]$ while clinically significant cases formed only $4,5 \%, 95 \% \mathrm{CI}[0,2 ; 22,8]$.

Conclusion: Augmentation with allograft bone particles provides stability of pedicle screw fixation in patients with severe osteoporosis. The suggested augmentation technique can be an alternative to PMMA augmentation as far no liquids are used. An additional benefit of the suggested solution is that also pedicle augmentation is available.

Keywords: Osteoporosis; Pedicle Screw; Allogenic Bone Graft; Traumatic Injuries; Polymethylmethacrylate; Thoracolumbar Junction; Fluoroscopy

\section{Introduction}

Osteoporosis is a frequently accounting condition in an elderly-adult population that is associated with decrease in bone mass and disruption of bone architecture that finally results in a load bearing capacity decrease [1]. Pedicle screw fixation is a frequently employed technique for degenerative diseases and unstable traumatic injuries treatment however this type of surgery remains a challenge in patients with osteoporosis as far as loosening rate may exceed $50 \%$ in those cases [2]. Taking into account risks of implant instability in patients with poor bone quality it is essential to achieve a sufficient purchase of screws in bone to resist physiological loads and to provide sufficient stability for fusion formation. Several strategies were suggested to achieve an increase in pedicle screw fixation strength in patients with poor bone quality and the most frequently used are based on bone cement augmentation [3,4]. Augmentation with polymethylmethacrylate (PMMA) and calcium phosphate are the most frequently used in clinical practice and it has been estimated that the former provide superior stability compared to the latter $[3,4]$. Even though PMMA provides substantial increase in screw fixation strength in bone, application of this technique is associated with a considerable rate of complications that are linked to the use of liquid cement and its chemical specificities. The most hazardous of those reported are intracanal cement leakage and pulmonary embolism. The reported complication rate ranges from $22,9 \%$ to $41,3 \%$ for the former and from 4,1 to 28,6 for the latter [5-8]. Other concerns associated with PMMA application are exothermic reaction of PMMA polymerization and the so-called bone cement implantation syndrome. It has been reported that heat release during PMMA polymerization can damage nerve roots and spinal cord finally causing persistent 
neurological deficit $[9,10]$. Cement implantation syndrome is an additional potential complication which pathophysiology is still debating. This adverse event can be manifested with hypotension, cardiac failure and even unexpected death in the perioperative period [11]. An additional disadvantage of cement augmentation is that it alters biomechanics of pedicle screw fixation favoring proximal loosening [12]. Considering all potential hazards associated with PMMA application safer alternatives that provide comparable biomechanical results are required.

\section{Materials and Methods}

This is a prospective non-randomized study of 28 patients with either unstable traumatic injuries of lumbar spine or thoracolumbar junction or spinal stenosis with unstable segments. All patients presented with severe osteoporosis with $\mathrm{T}$ criterion by DXA below $-3,5$ and radiodensity below $80 \mathrm{HU}$. All patients were treated employing pedicle screw fixation with allogeneic bone augmentation. Minimal follow-up period accounted for 18 months. This study was reviewed and approved and by local IRB committee. Patients underwent spinal instrumentation during the period from 2017 to 2018.

\section{The inclusion criteria}

a. Either unstable traumatic injury of lumbar spine or thoracolumbar junction - OF4, OF5 injuries according to classification of osteoporotic thoracolumbar fractures or degenerative stenosis of the lumbar spine with neurogenic claudication or radiculopathy with proven segmental instability.

b. For patients with degenerative diseases indications for surgery were intractable pain resistant to conservative treatment including blocks under fluoroscopy with VAS over 40 , ODI>40\% with neurogenic claudication or progressive neurological deficit.

c. Osteoporosis with Tcr below 3,5 estimated by DXA and radiodensity by CT below $80 \mathrm{HU}$.

\section{The exclusion criteria}

a. patients with more than 2 level degenerative stenosis that require spinal instrumentation

b. evidence of tumor-related lesions of the lumbar spine and thoracolumbar junction.

c. inflammatory diseases of the lumbar spine and thoracolumbar junction

d. patients with sagittal and frontal imbalance and spinopelvic parameters mismatches that require correction.

e. Pedicle diameter less than $6 \mathrm{~mm}$.

Before surgery, patients with degenerative diseases underwent X-ray imaging with flexion and extension to detect instability, all patients underwent CT examination and DXA. The criterion for spinal instability was anterior translation greater than $3 \mathrm{~mm}$ and rotation more than $10^{\circ}$ [13]. CT was used as a part of preoperative work in all patients. The CT scans were performed from the T12-L5 levels using a single CT scanner (Aquilion 32, Toshiba Corporation). The scans used a slice thickness of 0.5 $\mathrm{mm}$, covering a scan area of $50 \mathrm{~cm}$. The scan parameters were tube voltage $120 \mathrm{kV}$, tube current $300 \mathrm{~mA}$, auto $\mathrm{mAs}$ range 180 to 400; helical-pitch 21.0. Software utilized for calculations of bone density was Vitrea Version 5.2.497.5523 incorporating a window width/window level ratio of 2000/500. During CT examinations, measurements of a vertebral body cancellous bone radiodensity in HU were obtained at standard level of L3 or L2 in the sagittal, axial, and coronal planes. Measurements in the axial plane were taken at the level of the middle of the pedicles while those in the sagittal and coronal planes were taken along the geometric center of vertebra body. Out of those figures, mean radiodensity was calculated for each case. Patients with radiodensity below $80 \mathrm{HU}$, $\mathrm{Tcr}<-3,5$ were selected for this study.

Patients with degenerative stenosis and instability underwent open pedicle screw fixation with TLIF. The applied technique was standard: posterior decompression was performed with unilateral facet joint removal to approach disk that subsequently was removed totally. Once disc preparation had been complete TLIF with one bullet shape cage was performed. Finally pedicle screw fixation was performed applying direct trajectory parallel to the endplates with the suggested technique of vertebra augmentation. Patients with traumatic injuries underwent bisegmental pedicle screw fixation with additional screw introduced into fractured vertebrae and posterior fusion. In case of evident ligament disruption (OF5 injury) and considerable kyphotic deformity exceeding $10^{\circ}$ anterior reconstruction was performed using a mesh with allograft.

The applied technique for vertebra augmentation was the following: prior to screw insertion channels were created through pedicles into vertebral bodies using bipedicular approach. Then 5 $\mathrm{mm}$ bone funnel was introduced into a vertebral body. After that allograft particles were impacted into vertebral bodies through the funnel forming a hyperdense area on screw trajectory. Once vertebral body augmentation had been accomplished pedicles were filled up with allograft particles while removing the funnel. After augmentation had been completed again channels were created through pedicles into vertebral bodies and finally pedicle screws were introduced. Augmentation was performed bilaterally on each level excluding fractured vertebra in patients with osteoporotic fractures.

The follow-up period accounted for 18 months. Patients underwent examination in 3, 6, 12, 18 months after surgery. At 3 months patients were given X-Ray examination, CT was performed optionally if implant related complication was suspected. CT examination was applied in 6, 12, 18 months. During all visits, patients underwent investigation applying VAS ODI questionnaire and neurological examination. Cases with implant instability were 
registered. Criterion for pedicle screw loosening was at least $1 \mathrm{~mm}$ radiolucent zone around a screw surrounded by osteosclerotic rim [14]. Taking into account that clinical evidence of radiologic signs of loosening are limited, instability was considered clinically significant if a patient deteriorated with axial pain VAS over 40 and ODI $>40 \%$, if neither anterior nor posterior fusion was achieved according CT results or increase in local kyphosis was detected.

\section{Statistical Analysis}

The rate of postoperative complications with 95\% CI was calculated. No comparative analysis was performed.

\section{Results}

A heterogeneous group of 28 patients with degenerative diseases of the lumbar spine who underwent spinal instrumentations with pedicle screws fixation was enrolled in this study, the loss to follow-up accounted 6 patients (21\%), finally the results in 22 patients were studied. Characteristics of the patients are provided in (Table 1). According to the postoperative CT intracanal migration of material used for vertebrae augmentation were detected in 2 cases. The cause of material migration was pedicle breach during augmentation; all those cases were asymptomatic, therefore, no revision surgery was applied. No cases with pedicle screw fixator breakage were detected during the follow-up period (neither rods no screws). During the followup period CT signs of screw loosening were detected in 3 cases while the other types of instability were not detected. Out of those cases 2 were presented with a radiolucent zone around the screws more than $1 \mathrm{~mm}$. On the other hand no pain exacerbation was detected furthermore either anterior or posterior fusion was detected. Those cases were considered asymptomatic; therefore, no revision surgery was applied. One patient presented with clinically significant pedicle screw fixation instability that was detected 4 months after surgery. This complication presented with the increase in pain intensity that was the reason for an unplanned visit. CT was administered and pedicle screw instrumentation instability caused by windshield wiper effect was evaluated. This was the only patient who underwent revision surgery because of pedicle screw fixation failure.

Table1: Demographic characteristics of the enrolled group of patients.

\begin{tabular}{|c|c|}
\hline Number of cases & 22 \\
\hline Age & 62,$86 ; \mathrm{SD}=9,71 ; \min 39, \max 78$ \\
\hline Male to female ratio & $7 / 15$ \\
\hline Degenerative diseases & $5(22,7 \%)$ \\
\hline Traumatic injuries & $17(77,3 \%)$ \\
\hline
\end{tabular}

A considerable axial pain relief was evaluated in 21 case, no cases with pain intensity over 4 (VAS) and ODI over $40 \%$ were detected. No resorption of material used for screw augmentation was detected during the follow-up period, augmented parts of vertebrae remained hyperdense. Application of the suggested technique can be demonstrated by a clinical case. The patient was treated because of osteoporotic OF4 fracture of L4 vertebra. The calculated radiodensity came to $65 \mathrm{HU}$, the T criterion by DXA value accounted for -3,5. Patient underwent L3-L4-L5 pedicle screw fixation with suggested technique of augmentation. As only axial pain was present and no neurological symptoms were detected nerve root decompression was not performed. Preoperative results of CT examination are present in (Figures 1 \& 2). Postoperative CT images and results of CT examination 18 months after are presented in (figures 3-6) respectively.

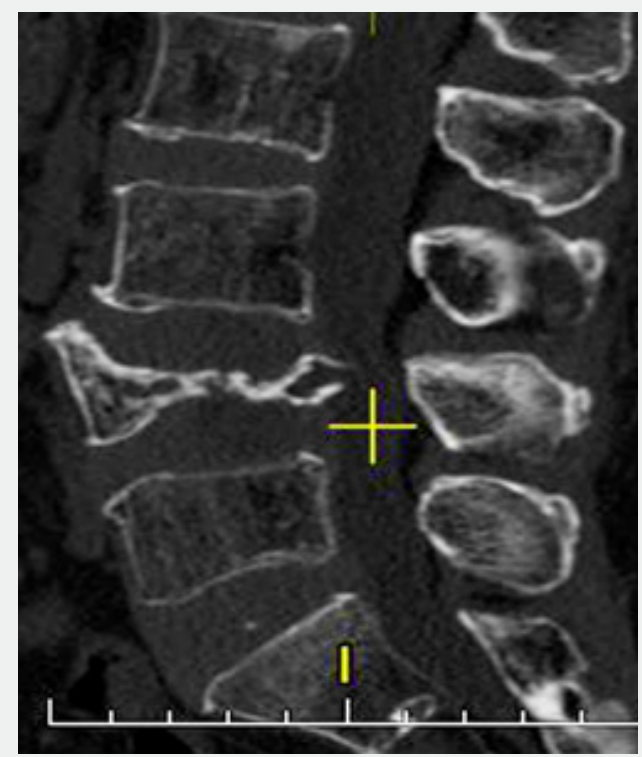

Figure 1: The result of preoperative CT examination, image in a sagittal plane. 


\section{Orthopedics and Rheumatology Open Access Journal (OROAJ)}

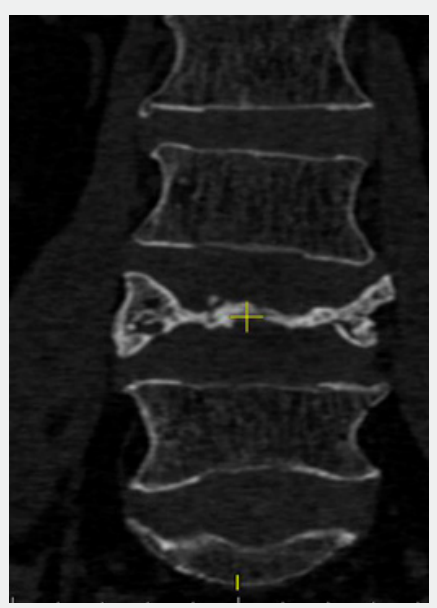

Figure 2: The result of preoperative CT examination, image in a coronal plane.

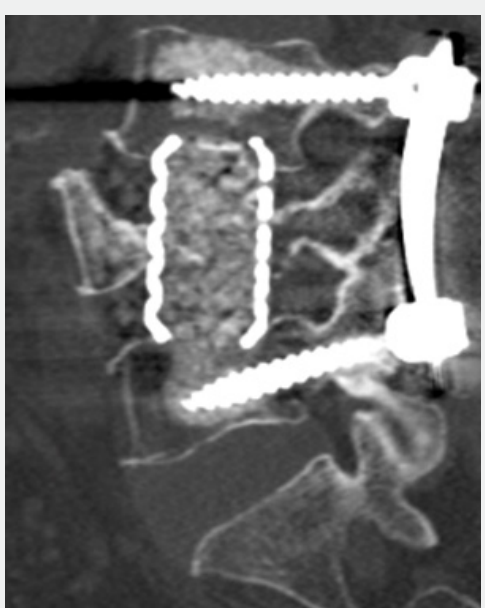

Figure 3: The result of postoperative CT examination, image in a sagittal plane.

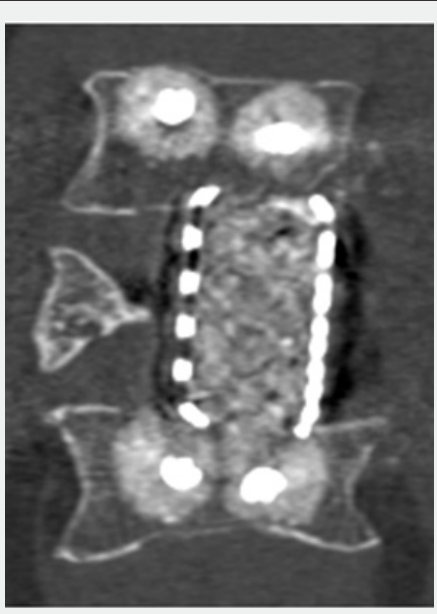

Figure 4: The result of postoperative CT examination, image in a coronal plane.

Finally, the suggested technique for pedicle screw augmentation provided pedicle screw fixation stability in patients with severe osteoporosis with the rate of instrumentation failure detected on CT accounting for $13,6,95 \%$ CI $[2,9 ; 34,9]$. Out of those clinically significant cases that required revision surgery formed only $4,5 \%, 95 \%$ CI $[0,2 ; 22,8]$. The achieved results demonstrate that augmentation using allograft particles is a suitable option for patients with severe osteoporosis which require pedicle screw fixation. 


\section{Orthopedics and Rheumatology Open Access Journal (OROAJ)}

\section{Discussion}

Being a considerable risk factor for pedicle screw loosening, osteoporosis strongly affects the results of pedicle screw fixation in elderly adult patients $[2,15,16]$. Different techniques were suggested to increase stability of pedicle screw fixation including modifications of a screw design, expandable screws and augmentation with PMMA or calcium phosphate cement. Out of those listed augmentation with PMMA was considered the most effective because it is capable of increasing pullout strength of pedicle screws 28 - 348 fold depending on the applied augmentation technique and the amount of cement $[3,4]$.

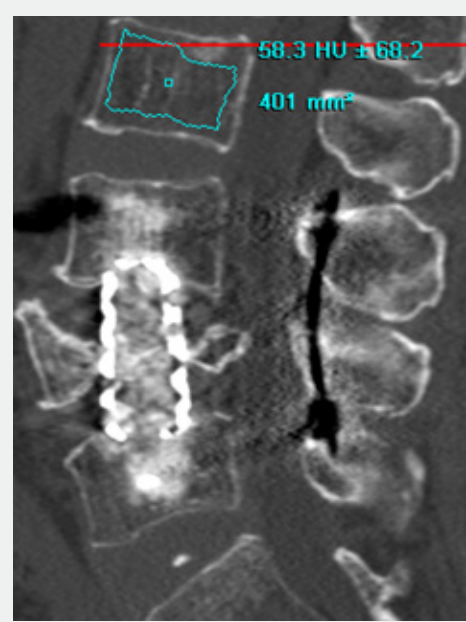

Figure 5: The result of postoperative CT examination taken during the follow-up period at 18th month after surgery, image in a sagittal plane.

Even though augmentation with PMMA demonstrated the best results in biomechanical tests, several controversies in its assessment are still present. The source of the reported incongruences is that the most frequently used tests in biomechanical works were pullout tests. It has been proven that a pullout mechanism causes a tiny minority of cases with pedicle screw fixation instability while the most frequent is mediated by craniocaudal toggling under physiological loads $[17,18]$. According to the results of biomechanical tests, the effectiveness of PMMA augmentation is greater in terms of pullout resistance while the effect on performance in fatigue tests is lower $[17,19]$. The observed differences can be explained by the fact that augmentation with PMMA alters biomechanics of pedicle screw fixation in shifting pivot point of the screw ventrally while pedicle provides at least $60 \%$ strength of pedicle screw fixation if a conventional technique is used [20]. Ventral shift of screw pivot point results in a different pattern of screw loosening - proximal loosening that is maximally presented in pedicles [12,21]. The way to overcome the discussed biomechanical drawback is augmentation of the whole screw trajectory including pedicles that is associated with an unacceptable risk if PMMA is used for augmentation [18].

Other issues related to PMMA application for screw augmentation are associated with various types of cement leakage that are capable to cause nerve root damage and pulmonary embolism. Nerve root damage can be caused not only by mass effect of extruded cement but also by liberation of heat during PMMA polymerization $[9,10]$. The reported rate of intracanal leakage is scattering between 22,9 and $41,3 \%$ while out of those cases asymptomatic ones account for 80-96,7 \% [5,6,8]. Even though the majority of patients with pulmonary embolism are asymptomatic, lethal cases were also reported, highlighting the existent hazards associated with the applied technique [8]. Regardless of the type of surgery, augmentation with PMMA has a potential danger of the so-called cement implantation syndrome development that is associated with cardiac failure and sudden death after surgery $[11,22,23]$. The potential mechanism of this complication is still debated, however diffusion of MMA monomer into blood circulation is considered one of potential reasons [11]. Taking into account risks associated with PMMA augmentation application of a safer technique is required to avoid the discussed risks.

Since 2016 augmentation with allograft bone particles has been applied in our clinic to increase pedicle screw fixation strength in patients with poor bone quality. The biomechanical study of similar technique give the evidence that augmentation with bone allograft particles provides a considerable increase in screw purchase strength in bone furthermore this study highlights benefits from pedicle augmentation [18]. In our study a slightly different modification is used as far as a greater hyperdense area is formed within a vertebral body that potentially strengthens pedicle screw fixation. The suggested technique provides favorable results in patients with severe osteoporosis compared to those achieved with PMMA augmentation application. The potential danger of the applied augmentation is a breach of pedicle with migration of allograft particles into a channel. To avoid this complication beforehand pedicle augmentation the integrity of internal cortical should be checked. During the follow- 
up period that accounted for 18 months the augmented area of vertebral body remained hyperdense without signs of allograft resorption. This study is limited by several factors; firstly a small group was enrolled however every new technique should be tested on small group with minimal risk of technique application. The enrollment of each patient was reviewed by the institutional board and application of PMMA was considered more hazardous than the suggested technique. This study may have a bias caused by heterogeneity of the enrolled group.

\section{Conclusion}

Augmentation with allograft bone particles provides stability of pedicle screw fixation in patients with severe osteoporosis. The suggested augmentation technique can be an alternative to PMMA augmentation as far no liquids are used. An additional benefit of the suggested solution is that also pedicle augmentation is available.

\section{References}

1. Cook SD, Salkeld SL, Stanley T, Faciane A, Miller SD (2004) Biomechanical study of pedicle screw fixation in severely osteoporotic bone. Spine J 4(4): 402-408.

2. Röllinghoff M, Schlüter BK, Groos D, Rolf S, Joern W, et al. (2010) Midrange outcomes in 64 consecutive cases of multilevel fusion for degenerative diseases of the lumbar spine. Orthop Rev (Pavia) 2(1): e3.

3. Shea TM, Laun J, Gonzalez BSA, James J, William E, et al. (2014) Designs and Techniques That Improve the Pullout Strength of Pedicle Screws in Osteoporotic Vertebrae: Current Status. Biomed Res Int 2014: 1-15.

4. Singh V, Mahajan R, Das K, Chhabra HS, Rustagi T (2019) Surgical Trend Analysis for Use of Cement Augmented Pedicle Screws in Osteoporosis of Spine: A Systematic Review (2000-2017). Glob Spine ] 9(7): 783-795.

5. Guo H, Tang Y, Guo D, Shun C, Yong X, et al. (2019) The cement leakage in cement-augmented pedicle screw instrumentation in degenerative lumbosacral diseases: a retrospective analysis of 202 cases and 950 augmented pedicle screws. Eur Spine J 28(7): 1661-1669.

6. Mueller JU, Baldauf J, Marx S, Kirsch M, Schroeder HWS, et al. (2016) Cement leakage in pedicle screw augmentation: a prospective analysis of 98 patients and 474 augmented pedicle screws. J Neurosurg Spine 25(1): 103-109.

7. Krueger A, Bliemel C, Zettl R, Ruchholtz S (2009) Management of pulmonary cement embolism after percutaneous vertebroplasty and kyphoplasty: a systematic review of the literature. Eur Spine J 18(9): 1257-1265.

8. Janssen I, Ryang YM, Gempt J, Bette S, Julia G, et al. (2017) Risk of cement leakage and pulmonary embolism by bone cement-augmented pedicle screw fixation of the thoracolumbar spine. Spine J 17(6): 837844.
9. Lai PL, Tai CL, Chen LH, Nien NY (2011) Cement leakage causes potential thermal injury in vertebroplasty. BMC Musculoskelet Disord 12: 116.

10. Sidhu GS, Kepler CK, Savage KE, Eachus B, Albert TJ, et al. (2013) Neurological deficit due to cement extravasation following a vertebral augmentation procedure. J Neurosurg Spine 19(1): 61-70.

11. Donaldson AJ, Thomson HE, Harper NJ, Kenny NW (2009) Bone cement implantation syndrome. Br J Anaesth 102(1):12-22.

12. Choy WJ, Walsh WR, Phan K, Mobbs RJ (2019) Technical Note: Pedicle Cement Augmentation with Proximal Screw Toggle and Loosening. Orthop Surg 11(3): 510-515.

13. Leone A, Guglielmi G, Cassar PVN, Bonomo L (2007) Lumbar Intervertebral Instability: A Review. Radiology 245(1): 62-77.

14. Galbusera F, Volkheimer D, Reitmaier S, Berger RN, Kienle A, et al. (2015) Pedicle screw loosening: a clinically relevant complication? Eur Spine J 24(5): 1005-1016.

15. Sandén B, Olerud C, Larsson S, Robinson Y (2010) Insertion torque is not a good predictor of pedicle screw loosening after spinal instrumentation: a prospective study in 8 patients. Patient Saf Surg 4(1): 14.

16. Rometsch E, Spruit M, Zigler JE, Venugopal KM, Jean AO, et al. (2020) Screw-Related Complications After Instrumentation of the Osteoporotic Spine: A Systematic Literature Review With Meta-Analysis. Glob Spine J 10(1): 69-88.

17. Kueny RA, Kolb JP, Lehmann W, Püschel K, Morlock MM, et al. (2014) Influence of the screw augmentation technique and a diameter increase on pedicle screw fixation in the osteoporotic spine: pullout versus fatigue testing. Eur Spine J 23(10): 2196-2202.

18. Jia C, Zhang R, Xing T, Huimin L, Fulong D, et al. (2019) Biomechanical properties of pedicle screw fixation augmented with allograft bone particles in osteoporotic vertebrae: different sizes and amounts. Spine J 19(8): 1443-1452.

19. Bostelmann R, Keiler A, Steiger HJ, Scholz A, Cornelius JF, et al. (2017) Effect of augmentation techniques on the failure of pedicle screws under cranio-caudal cyclic loading. Eur Spine J 26(1): 181-188.

20. Wadhwa RK, Thakur JD, Khan IS, Imad SK, James Jet al. (2015) Adjustment of Suboptimally Placed Lumbar Pedicle Screws Decreases Pullout Strength and Alters Biomechanics of the Construct: A Pilot Cadaveric Study. World Neurosurg 83(3): 368-375.

21. Weiser L, Huber G, Sellenschloh K, Lennart Vet, Klaus P, et al. (2018) Time to augment?! Impact of cement augmentation on pedicle screw fixation strength depending on bone mineral density. Eur Spine J 27(8): 1964-1971.

22. Jenkins K, Wake PJ (2002) Cement implantation syndrome. Anaesthesia. 57(4): 404-418.

23. Sawakami K, Yamazaki A, Ishikawa S, Ito T, Watanabe K, et al. (2012) Polymethylmethacrylate Augmentation of Pedicle Screws Increases the Initial Fixation in Osteoporotic Spine Patients. J Spinal Disord Tech 25(2): E28-E35. 


\section{Your next submission with Juniper Publishers} will reach you the below assets

- Quality Editorial service

- Swift Peer Review

- Reprints availability

- E-prints Service

- Manuscript Podcast for convenient understanding

- Global attainment for your research

- Manuscript accessibility in different formats ( Pdf, E-pub, Full Text, Audio)

- Unceasing customer service

Track the below URL for one-step submission https://juniperpublishers.com/online-submission.php 